\title{
The impact of COVID-19 on routine patient care from a laboratory perspective
}

\author{
E C Kruger, MB ChB, FC Path (Chem), MMed (Chem Path); R Banderker, MB ChB; \\ R T Erasmus, MBBS, FMC Path, FACB, DABCC, DHSM; A E Zemlin, MB ChB, FC Path (Chem), MMed (Chem Path), PhD
}

Division of Chemical Pathology, Department of Pathology, National Health Laboratory Service, Tygerberg Hospital and Faculty of Medicine and Health Sciences, Stellenbosch University, Cape Town, South Africa

Corresponding author: E C Kruger(elsie.kruger@nhls.ac.za)

Background. Globally, few studies have examined the effect of the COVID-19 pandemic on routine patient care and follow-up.

Objectives. To evaluate the effect of the COVID-19 response on biochemical test requests received from outpatient departments (OPDs) and peripheral clinics serviced by the National Health Laboratory Service Chemical Pathology Laboratory at Tygerberg Hospital, Cape Town, South Africa (SA). Request volumes were used as a measure of the routine care of patients, as clinical information was not readily available.

Methods. A retrospective audit was conducted. The numbers of requests received from OPDs and peripheral clinics for creatinine, glycated haemoglobin (HbAlc), lipid profiles, thyroid-stimulating hormone (TSH), free thyroxine, free tri-iodothyronine (fT3), serum and urine protein electrophoresis, serum free light chains and neonatal total serum bilirubin were obtained from 1 March to 30 June for 2017,2018 , 2019 and 2020.

Results. The biggest impact was seen on lipids, creatinine, HbA1c, TSH and fT3. The percentage reduction between 1 March and 30 June 2019 and between 1 March and 30 June 2020 was 59\% for lipids, 64\% for creatinine and HbA1c, 80\% for TSH and 81\% for fT3. There was a noteworthy decrease in overall analyte testing from March to April 2020, coinciding with initiation of level 5 lockdown. Although an increase in testing was observed during June 2020, the number of requests was still lower than in June 2019.

Conclusions. This study, focusing on the short-term consequences of the SA response to the COVID-19 pandemic, found that routine follow-up of patients with communicable and non-communicable diseases was affected. Future studies are necessary to evaluate the longterm consequences of the pandemic for these patient groups.

S Afr Med J 2020;110(12):1201-1205. https://doi.org/10.7196/SAMJ.2020.v110i12.15294

The World Health Organization (WHO) officially declared COVID19 a pandemic on 11 March $2020,{ }^{[1]}$ and it has had far-reaching effects on both the international and local fronts. At the time of writing (10 September 2020) there had been close to 28 million confirmed cases worldwide, with the death toll over $900000 .{ }^{[2]}$ On 23 March, the South African (SA) government announced a strict nationwide level 5 lockdown. At Tygerberg Hospital (TBH) in Cape Town, as in most hospitals globally, many outpatient departments (OPDs) were closed as routine patient follow-up visits and elective surgeries were postponed. Furthermore, many healthcare workers were redeployed to serve in the front line, preventing them from delivering specialised care. Several peripheral clinics were closed for short periods at a time, possibly to allow decontamination of the facilities following confirmation of COVID-19 cases, resulting in patients not being able to access their usual care. Some patients may not have attended their follow-up visits for fear of contracting the virus.

In SA, both communicable (mainly tuberculosis and human immunodeficiency virus (HIV)) and non-communicable diseases (NCDs) contribute to the burden of disease. Globally, there have been few studies examining the effect of the COVID-19 pandemic on routine patient care and follow-up. To our knowledge, there are only three such studies in $\mathrm{SA},{ }^{[3-5]}$ and none specifically looking at the effect on biochemical laboratory tests.

\section{Objectives}

Since the lockdown was introduced in SA, the laboratory staff at the National Health Laboratory Service (NHLS) Chemical Pathology Laboratory have subjectively observed a decline in the number of routine chemistry requests received, as well as in income generated. The objective of this study was to examine the effect of the COVID19 response on the routine care of patients followed up at OPDs and peripheral hospitals that are serviced by the NHLS Chemical Pathology Laboratory at TBH. Since clinical and medication information was not available, laboratory request volumes were audited over a 4-month period after the initiation of hard lockdown. Patient followup was examined in four areas: HIV-infected subjects receiving antiretroviral therapy (ART), NCDs, malignancy (haematological and thyroid), and neonatal care.

\section{Methods \\ Study design}

This descriptive (observational) study was conducted at the NHLS Chemical Pathology Laboratory, TBH. TBH is a tertiary teaching hospital affiliated to Stellenbosch University and was the dedicated COVID-19 referral centre for Western Cape Province during the pandemic. ${ }^{[3]}$ As this was a laboratory study and no clinical or medication information was available, laboratory results were used as a proxy for patient follow-up. Data were extracted from the NHLS laboratory information system (LabTrak) from 1 March to 30 June for 2017, 2018, 2019 and 2020. We examined the following as they related to the general burden of disease and to some extent the paediatric population: (i) ART follow-up: all requests received for creatinine follow-up from ART clinics; (ii) NCD follow-up: glycated haemoglobin $(\mathrm{HbAlc})$ and lipid profile requests from OPDs and peripheral clinics, and free thyroxine (fT4) requests from peripheral clinics for patients on levothyroxine therapy; (iii) cancer follow-up: 
thyroid-stimulating hormone (TSH) and free tri-iodothyronine (fT3) requests for patients receiving radioactive iodine treatment, and all serum and urine protein electrophoresis (SPEP, UPEP) and serum free light chain (SFLC) requests from haematology clinics; and (iv) neonatal care: neonatal total serum bilirubin requests from midwife obstetric units (MOUs) and peripheral clinics.

\section{Ethical considerations}

Patient confidentiality was ensured by labelling data with a unique laboratory episode number. No patient recruitment was required. The only demographic information recorded was the requesting facility. Only the principal investigator and co-investigators had access to patient sample information. The study was approved by the Ethics Committee of Stellenbosch University (ref. no. N20/07/035 CIVID-19), and the research was conducted according to the ethical principles of the Declaration of Helsinki (2013).

\section{Data analysis}

Excel (Office 365; Microsoft, USA) was used to analyse data and to construct graphs. Results were grouped and tabulated according to analyte, and the month, year and site of collection. The number of requests per analyte was compared between each year of the study, and the percentage difference was calculated. The trend of requests for each month of the study in 2020 was evaluated and compared with the trend of requests for the same period in 2019.

\section{Results}

During the 4-month study period (1 March - 30 June) in 2020, there was a clear decrease in the number of requests received for all analytes included in the audit, with the exception of SFLC and neonatal total bilirubin, when compared with the same period in 2017, 2018 and 2019 (Table 1).

The biggest impact was seen on lipids, creatinine, HbAlc, TSH and fT3. The percentage difference between 1 March - 30 June 2020 and 1 March - 30 June 2019 was 59\% for lipids and 64\% for creatinine and $\mathrm{HbA1c}$, while for TSH and fT3 it was $80 \%$ and $81 \%$, respectively.

Although the number of neonatal total bilirubin requests remained unchanged, the number of clinics from which the laboratory received these requests decreased (from 27 in 2019 to 16 in 2020).

Unexpectedly, a downward trend in creatinine requests was noted in each consecutive year from 2017 to 2019. Lipid profile, TSH, fT4 and fT3 requests decreased in 2018 (relative to 2017), with a subsequent increase in 2019.

There was a noteworthy decrease in overall analyte testing from March to April 2020 (Fig. 1). Fig. 1 shows a significant decrease in April - this coincides with initiation of the level 5 lockdown. Although an increase in testing was observed during June 2020 in line with the easing of lockdown to levels 4 and then 3 , the number of requests was still lower compared with the number of requests in June 2019 (Table 2).

\section{Discussion}

The objective of our study was to determine the impact of SA's COVID-19 lockdown strategy on the routine follow-up of patients with communicable diseases and NCDs, specifically patients receiving ART, patients being treated for diabetes, dyslipidaemia and hypothyroidism, and patients with malignancies (haematological and thyroid), and on neonatal follow-up. We used the laboratory

Table 1. Number of requests received from 1 March to 30 June for $2017-2020$

\begin{tabular}{|c|c|c|c|c|c|c|c|}
\hline \multirow[b]{2}{*}{ Analyte } & \multirow[b]{2}{*}{2017} & \multirow[b]{2}{*}{2018} & \multirow[b]{2}{*}{2019} & \multirow[b]{2}{*}{2020} & \multicolumn{3}{|c|}{ Difference, $\%$} \\
\hline & & & & & 2017 and 2018 & 2018 and 2019 & 2019 and 2020 \\
\hline Neonatal total bilirubin & 649 & 1112 & 1234 & 975 & 71 & 11 & -21 \\
\hline Creatinine & 35113 & 34442 & 10323 & 3713 & -2 & -70 & -64 \\
\hline $\mathrm{HbAlc}$ & 5861 & 6162 & 12120 & 4341 & 5 & 97 & -64 \\
\hline Lipids & 1629 & 926 & 1750 & 726 & -43 & 89 & -59 \\
\hline SFLC & 94 & 158 & 370 & 477 & 68 & 134 & 29 \\
\hline SPEP & 812 & 698 & 354 & 341 & -14 & -49 & -4 \\
\hline TSH & 369 & 311 & 341 & 67 & -16 & 10 & -80 \\
\hline fT4 & 1981 & 1923 & 3583 & 1651 & -3 & 86 & -54 \\
\hline fT3 & 365 & 308 & 334 & 62 & -16 & 8 & -81 \\
\hline UPEP & 245 & 194 & 117 & 79 & -21 & -40 & -32 \\
\hline
\end{tabular}

$\mathrm{HbAlc}$ = glycated haemoglobin; $\mathrm{SFLC}=$ serum free light chains; SPEP = serum protein electrophoresis; TSH = thyroid-stimulating hormone; fT4 = free thyroxine; $\mathrm{fT} 3$ = free tri-iodothyronine; HPAIC $=$ glycated haemoglobin,
$\mathrm{UPEP}=$ urine protein electrophoresis.

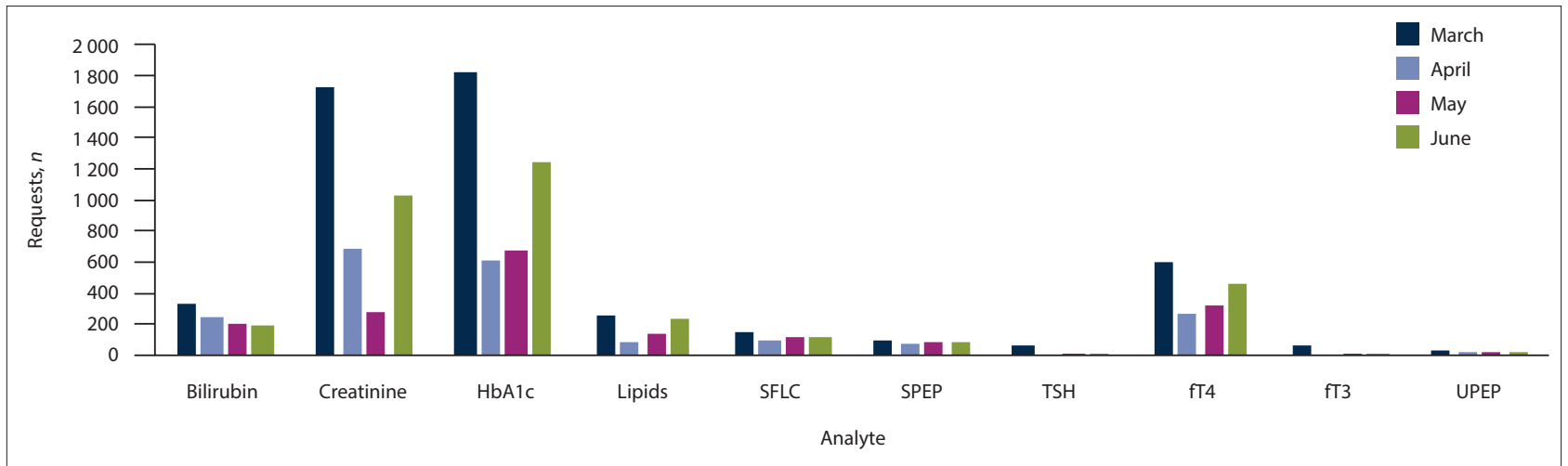

Fig. 1. Number of test requests per month during March - June 2020. (HbA1c = glycated haemoglobin; SFLC = serum free light chains; SPEP = serum protein electrophoresis; $T S H=$ thyroid-stimulating hormone; $f T 4=$ free thyroxine; $f T 3=$ free tri-iodothyronine; UPEP = urine protein electrophoresis.) 


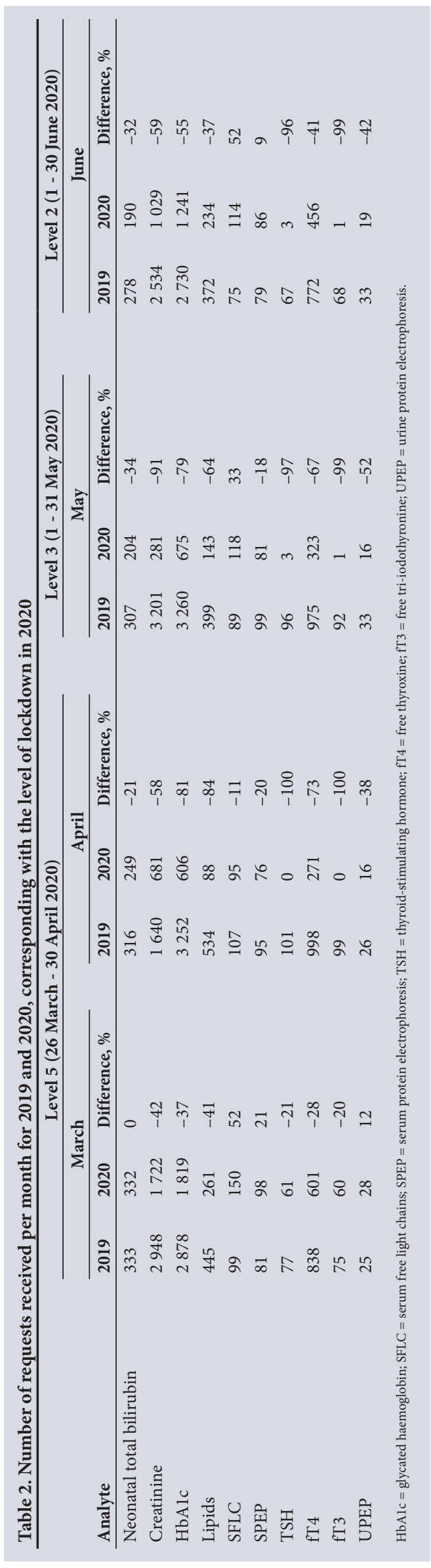

tests usually requested in the monitoring of these conditions as a proxy of patient follow-up.

We noted a distinct decrease in the relevant testing, particularly in April 2020, which coincides with initiation of the stage 5 lockdown. This was especially relevant for HbAlc (diabetes follow-up), creatinine from ART clinics, and thyroid function tests (thyroid malignancy and hypothyroidism follow-up). The follow-up of haematological malignancies and neonatal bilirubin was least affected.

During the past 20 years, considerable progress has been made in the fight against HIV/AIDS, as the rate of new infections decreased by $39 \%$ and the number of deaths related to HIV fell by $51 \%{ }^{[6]}$ This progress is threatened by treatment interruptions. Creatinine is routinely measured during the follow-up of patients receiving ART. We found that there was a $64 \%$ reduction in requests received in 2020 compared with 2019. However, creatinine also decreased between 2018 and 2019 , which could possibly be due to the introduction of point-of-care creatinine testing in the clinics. We postulate that the reduction seen between 2019 and 2020 was due to the lockdown. Madhi et al. ${ }^{[4]}$ also investigated the effects of COVID-19 on patient follow-up and observed that the average HIV viral load testing conducted per week across SA decreased by $22 \%$ after lockdown implementation, while CD4+ count testing decreased by $33 \%$. A 6-month interruption in ARV treatment has the potential to increase the number of HIV-related deaths by 500 000. Five years from now, HIV mortality could increase by $40 \%{ }^{[7]}$ Vertical transmission, from mother to child, will also potentially double.

A study by Siedner et al. ${ }^{[5]}$ studied the effect of lockdown on primary healthcare clinic visits in the northern part of KwaZulu-Natal Province, comparing the number of visits prior to and during lockdown. They reported a 20\% increase in visits to ART clinics just before the implementation of lockdown, and postulated that this may have been an attempt to collect antiretrovirals in anticipation of an interruption in services. The Western Cape Department of Health is in the process of developing a model whereby ART medication is directly delivered to patients' homes. If blood samples are needed, a phlebotomist will be deployed. ${ }^{[8]}$

Annually, NCDs are responsible for 41 million deaths globally ${ }^{[9]}$ The pandemic has led to significant disruptions in the routine care of NCDs, with many follow-up visits postponed in an attempt to lessen the burden on hospitals. Healthcare workers from various departments have been redeployed to assist in the management of COVID-19 cases. $^{[10]}$ We found that the number of HbAlc requests, serving as a marker for the follow-up of diabetic patients, was $64 \%$ lower than in 2019 . We found an impressive increase in the number of $\mathrm{HbAlc}$ requests between 2018 and 2019 , and postulate that this was due to the introduction of routine HbA1c testing as a screening test for diabetes and test redistribution, with more requests being sent to our laboratory from the catchment area. Similarly, the number of lipid profile requests, a marker of dyslipidaemia follow-up, decreased by $59 \%$.

A survey completed by 47 countries confirmed a change in the management of patients with NCDs. Thirty-eight percent of respondents reported that diabetic patients were most affected. ${ }^{[11]} \mathrm{A}$ second survey, by the WHO, ${ }^{[9]}$ concluded that the impact of COVID-19 on NCDs is a global phenomenon; however, low-income countries are more affected. This survey reported that $49 \%$ of services for diabetes and related complications have been disrupted. ${ }^{\left[{ }^{[9]}\right.}$

SPEP, UPEP and SFLC are used in the monitoring of patients with monoclonal gammopathies. We noticed a sharp decrease in SPEP requests between 2018 and 2019, which coincides with the introduction of SPEP testing in Eastern Cape Province at this time and therefore a decrease in requests received at our laboratory. In comparison with 2019, our study showed a significant decrease in UPEP requests in 2020 (32\%), a marginal decrease in SPEP requests (4\%) and an increase in SFLC requests (30\%) from haematology clinics, suggesting that follow-up of these patients continued, even during the lockdown. Lack of patient access to devices with telecommunication capabilities could explain their ongoing clinic follow-up. Since 2019, SFLC analysis became part of the multiple myeloma monitoring profile at a neighbouring tertiary hospital, Groote Schuur Hospital, and the samples are referred to TBH. This may explain the increase in request volume compared with previous years.

TSH and fT 3 are routinely requested by the radioactive iodine ablation clinic (performed for the treatment of hyperthyroidism and thyroid carcinomas). These 
requests decreased dramatically from 2019 to 2020 (80\% and $81 \%$, respectively). A small study in Jordan ${ }^{[12]}$ evaluating the impact of COVID-19 on the treatment of thyroid cancer showed that surgical procedures were unaffected; however, adjunct treatment with radioactive iodine was altered. The change in treatment strategy led to additional financial costs to patients, and increased anxiety in some. ${ }^{[12]}$ The decrease in $\mathrm{fT} 4$ requests from peripheral clinics between 2019 and 2020 may be indicative of the suspension of routine followup of patients on levothyroxine due to the lockdown. The increase in fT4 that we noted between 2018 and 2019 is possibly due to referral of specimens from the peripheral clinics to our laboratory with a change in the catchment area.

The COVID-19 pandemic has affected the management of cancer patients in various ways. Many screening programmes have been halted, which is likely to lead to delayed diagnoses and adverse patient outcomes. ${ }^{[13]}$ A $72 \%$ reduction has been reported in the number of suspected cancer patients referred for further investigation by primary care physicians in Scotland, compared with previous weekly averages in the month of April. ${ }^{[14]}$

The effect of COVID-19 on neonatal care has not been well described. Neonatal total bilirubin was included as part of our study to evaluate the effect of the pandemic on the monitoring of neonatal jaundice. We illustrated that the number of requests in 2020 was consistent with requests in previous years; however, these requests were received from fewer clinics compared with 2019. The decreased number of clinics could be due to downscaling of obstetric services or closure of clinics during deep-cleaning procedures. Inevitably, patients who are normally served by the affected MOUs and clinics would have been redirected to other healthcare facilities, placing additional strain on an already overburdened system.

A survey in Oregon, USA, investigating changes in immunisation practices due to COVID-19, reported a 50\% rate of cancellation or rescheduling of immunisations. ${ }^{[15]}$ One of the concerns raised by clinics participating in the survey was the possible loss of follow-up of children who had missed immunisations. The decline in routine childhood vaccinations increases the risk of communicable diseases, ${ }^{[16]}$ such as measles, pertussis and rotavirus-associated gastroenteritis. ${ }^{[17]}$

As has been reported by others, we found that much of the focus on healthcare seems to have been directed to COVID-19. If access to certain types of care is reduced, people's health is likely to suffer. Indeed, a sharp increase in the number of natural deaths has been observed since the beginning of May across SA, the Western Cape and the Cape Town metro. ${ }^{[18]}$ A possible explanation for this increase could be that patients are refraining from seeking medical care. The Centers for Disease Control and Prevention in the USA reported a $20 \%$ decrease in emergency room visits for myocardial infarctions and CVAs compared with pre-pandemic figures. ${ }^{[19]} \mathrm{A} 10 \%$ reduction in visits for diabetic-related complaints was observed.

\section{Study limitations}

Although our study had several strengths, such as using a large number of tests reflecting both NCDs and communicable diseases spread over the past 3 years, it also had several limitations. The study looked at laboratory test requests as a proxy for routine patient care, and no clinical information was used to assess patient follow-up. We only investigated the impact on patient care by looking at requests received at a tertiary hospital laboratory in the public sector, and are therefore unable to report on the impact in private practice and at smaller laboratories. Clinical information is rarely provided on laboratory request forms, so it is possible that not all fT4 and HbAlc requests were for patients on levothyroxine or for diabetic follow-up, respectively. As this study only included tests performed at a chemical pathology laboratory, the impact on prominent communicable diseases such as tuberculosis and malaria could not be determined. It is likely that non-COVID-19 patient care has been affected to an equal extent in other parts of SA and Africa in general, but we are unable to confirm this. Another possible limitation is that we did not determine whether the reductions noted were due to the introduction of point-of-care testing by our clients. This is unlikely, however, as it would have taken time to introduce, and the instrument would have needed to be verified using laboratory results as a gold standard.

\section{Conclusions}

This study focused on the short-term healthcare consequences of the SA response to the COVID-19 pandemic. The long-lasting effects on the management of both communicable diseases and NCDs have yet to be established. In the immediate aftermath, the healthcare system in SA will be overwhelmed as it tries to relieve the backlog caused by suspended routine services, and in the long term it may be affected as it attempts to manage complications caused by delayed diagnoses.

The lack of follow-up during lockdown could result in the delayed detection of abnormal HbAlc and lipids, increasing risk for CVD. The suspension of clinical trials in cancer research has resulted in fewer possible treatment options for individuals who might otherwise have been included in these trials, and will also lead to delays in newer therapies becoming available to the public.

Telemedicine has been widely proposed as an alternative to physical interaction between patients and healthcare workers during these unprecedented times. While this novel form of patient care could be a welcome solution in developed countries, it is probably not feasible in much of Africa and other low-income countries.

We have been successful in limiting the potentially devastating effects of one pandemic. The next challenge will be to manage the possible surge of NCD complications, and a well-co-ordinated response will be just as important if we are to emerge victorious. Future studies are necessary to evaluate the consequences of delayed patient follow-up.

Declaration. None.

Acknowledgements. None.

Author contributions. ECK: data collection, data analysis, original and final draft writing; RB: data collection, original and final draft writing; RTE: literature review, proofreading article prior to submission; AEZ: conceptualised the study, assessment of findings, literature review and original and final draft writing, proofreading article prior to submission. Funding. None.

Conflicts of interest. None

1. World Health Organization. Archived: WHO Timeline - COVID-19. 27 April 2020. https://www.who. int/news-room/detail/27-04-2020-who-timeline---covid-19 (accessed 29 June 2020).

Johns Hopkins University \& Medicine. COVID-19 map - Johns Hopkins Coronavirus Resource Center. 2020. https://coronavirus.jhu.edu/map.html (accessed 10 September 2020).

3. Parker A, Karamchand S, Schrueder N, et al. Leadership and early strategic response to the SARS3. Parker A, Karamchand S, Schrueder N, et al. Leadership and early strategic response to the SARS-
CoV-2 pandemic at a COVID-19 designated hospital in South Africa. S Afr Med J 2020;110(6):463-

465. https://doi.org/10.7196/SAMJ.2020v110i6.14809 Success against COVID-19 at the price of greater costs. S Afr Med J 2020;110(8):724-726. https://doi. org/10.7196/SAMJ.2020.v110i8.15055

5. Siedner MJ, Kraemer JD, Meyer MJ, et al. Access to primary healthcare during lockdown measures for COVID-19 in rural South Africa: A longitudinal cohort study. medRxiv 2020 (epub 20 May 2020). https://doi.org/10.1101/2020.05.15.20103226

6. World Health Organization. WHO: Access to HIV medicines severely impacted by COVID-19 as AIDS response stalls. 6 July 2020. https://www.who.int/news/item/06-07-2020-who-access-to-hivmedicines-severely-impacted-by-covid-19-as-aids-response-stalls (accessed 16 August 2020).

Keene C, Mohr-Holland E, Cassidy T, et al. How COVID-19 could benefit tuberculosis and HIV services in South Africa. Lancet Respir Med 2020;8(9):844-846. https://doi.org/10.1016/S22132006(20)30311-8

8. Mukumbang FC, Kriel E, van Wyk B, et al. Desperate times call for desperate measures: Adapting antiretroviral service delivery in the context of the COVID-19 pandemic. S Afr Med J 2020;110(8):711712. https://doi.org/10.7196/SAMI.2020.v110i8.14967 
9. World Health Organization. COVID-19 significantly impacts health research for noncommunicable diseases. 1 June 2020. https://www.who.int/news-room/detail/01-06-2020-covid-19-significantlyimpacts-health-services-for-noncommunicable-diseases (accessed 16 August 2020).

10. Palmer K, Monaco A, Kivipelto M, et al. The potential long-term impact of the COVID-19 outbreak on patients with non-communicable diseases in Europe: Consequences for healthy ageing. Aging Clin Exp Res 2020;32(7):1189-1194. https://doi.org/10.1007/s40520-020-01601-4

11. Chudas A global survey of views from healthcare professionals. Diabetes Metab Syndr 2020;14(5):965-967. https://doi.org/10.1016/..dsx.2020.06.042

12. Bakker S, Al-Omar K, Aljarrah Q, et al. Impact of COVID-19 on thyroid cancer surgery and adjunct therapy. Updates Surg 2020;72:1-3. https://doi.org/10.1007/s13304-020-00833-3

13. Richards M, Anderson M, Carter P, Ebert BL, Mossialos E. The impact of the COVID-19 pandemic on cancer care. Nat Cancer 2020;1:565-567. https://doi:10.1038/s43018-020-0074-y

14. BBC News. Coronavirus in Scotland: Fears raised over fall in cancer case referrals. 20 April 2020. https://www.bbc.com/news/uk-scotland-52353657 (accessed 7 September 2020).

15. Robison S, Murray S, Luther M. Oregon survey of clinic immunization practice changes due to the COVID-19 pandemic. Oregon Health Authority, 5 May 2020. https://www.oregon.gov/oha/PH/ PREVENTIONWELLNESS/VACCINESIMMUNIZATION/Documents/Imm-COVIDsurvey.pdf (accessed 29 June 2020).
16. Santoli JM, Lindley MC, DeSilva MB, et al. Effects of the COVID-19 pandemic on routine pediatric vaccine ordering and administration - United States, 2020. MMWR Morb Mortal Wkly Rep 2020;69(19):591-593. https://doi.org/10.15585/mmwr.mm6919e2

17. Hungerford D, Cunliffe NA. Coronavirus disease (COVID-19) - impact on vaccin preventable diseases. Eurosurveillance 2020;25(18):2000756. https://doi.org/10.2807/1560-7917 ES.2020.25.18.2000756

18. South African Medical Research Council. Report on weekly deaths in South Africa. 2020. https:// www.samrc.ac.za/reports/report-weekly-deaths-south-africa?bc=254 (accessed 4 September 2020).

19. Lange SI, Ritchey MD, Goodman AB, et al. Potential indirect effects of the COVID-19 pandemic on use of emergency departments for acute life-threatening conditions - United States, January May 2020. MMWR Morb Mortal Wkly Rep 2020;69(25):795-800. https://doi.org/10.15585/mmwr $\mathrm{mm} 6925 \mathrm{e} 2$

Accepted 20 October 2020. 